\title{
The characteristics of patients with Acute Kidney Injury in Local Hospitals: A National Survey from China
}

\section{Lihua Wu}

General Hospital of Ningxia Medical University

Yaqi Ma

General Hospital of Ningxia Medical University

Jinwei Wang

Peking University First Hospital

\section{Fengwu Luan}

General Hospital of Ningxia Medical University

\section{Mengting Wang}

General Hospital of Ningxia Medical University

\section{Zhihong Feng}

General Hospital of Ningxia Medical University

\section{Yanhong Tian}

General Hospital of Ningxia Medical University

Lu Li

General Hospital of Ningxia Medical University

\section{Rui Chu}

General Hospital of Ningxia Medical University

Xiaolong Yin

General Hospital of Ningxia Medical University

Yan Wang

General Hospital of Ningxia Medical University

Yanmei Li

General Hospital of Ningxia Medical University

Xiaoyan Guo

General Hospital of Ningxia Medical University

Xiaoling Zhou

General Hospital of Ningxia Medical University

Min Yu

General Hospital of Ningxia Medical University

Xiaojuan Yang

Ningxia Medical University

Li Yang ( $\sim$ li.yang@bjmu.edu.cn )

Peking University First Hospital

Menghua Chen

General Hospital of Ningxia Medical University

\section{Research Article}

Keywords: Acute kidney injury, Local hospital, Economic status, Geographic regions, China

Posted Date: April 20th, 2021 
DOI: https://doi.org/10.21203/rs.3.rs-401479/v1

License: @ (1) This work is licensed under a Creative Commons Attribution 4.0 International License. Read Full License 


\section{Abstract}

Background冈Improving the diagnosis and treatment of acute kidney injury (AKI) in local hospitals is of great significance in developing countries. The aim of the present survey was to describe the characteristics of AKI in local hospitals at different economic levels and in different regions in mainland China.

Methods $₫$ We used the database of a nation-wide survey on AKI in mainland China and extracted the data from 22 local hospitals. These hospitals were divided into three groups based on the local per capita gross domestic product (pcGDP) levels. We also divided the hospitals by geographic regions into Northwest China, North China, South China, and Southwest China. Using this data, we analyzed all characteristics associated with AKI.

Results: 1,942 patients were diagnosed with AKI in the 22 local hospitals between January and July 2013, with a detection rate of $1.66 \%$. There were significant differences in the detection rates and the classification of AKI across regions with different economic levels or geographic locations. The proportion of patients who had not been recognized with AKI on time, or with unclassifed AKI was higher in local hospitals from regions with lowest and moderate pcGDP levels than that in regions with the highest pcGDP levels. Renal hypoperfusion, drug factors and associated with other serious diseases were the most common causal factors of AKI in all regions. Northwest and Southwest regions had higher proportion of patients with potential nephrotoxic drugs use. The Southwest region had the most patients with environmental toxin-induced AKI compared to other regions. AKI caused by sepsis or surgical factors was more common in South China. The all-cause in-hospital mortality was $10.5 \%$. Advanced age, male gender, staying in ICU, higher AKI stages, using nephrotoxic drugs, being with other critical illness, and delayed recognition were associated with increased inhospital mortality, while in hospitals from South China or from regions with the highest pcGDP levels were associated with decreased in-hospital mortality.

Conclusions: The detection rate of AKI in local hospitals in China is significantly lower than that in provincial hospitals. The etiology of AKI varies across different geographic regions and the level of economic development is closely related to the diagnosis of AKI in local hospitals.

\section{Background}

Acute kidney injury (AKI) can be caused by various etiologies. It is characterized by high mortality rates and progression to chronic kidney disease[1, 2]. In 2013, the International Society of Nephrology (ISN) launched a global goal which was referred to as '0by25'; in other words, the Society aimed to eliminate all deaths related to untreated acute kidney failure by 2025[3]. To facilitate this goal, a number of in-depth research studies have been conducted on AKI, thus enhancing the general awareness of this condition.

China is the world's largest developing country. Over recent years, China's economy has developed rapidly. However, regions and cities across China have developed increasing differences in terms of economy, culture, education, and medical care. Consequently, there is significant disparity between provincial and local hospitals with regards to the diagnosis and treatment of AKI. It has also been reported that the clinical features of AKI differ between low-income countries and high-income countries[4]. In our previous study, we observed significant differences in patient age, cause, and type of AKI, when compared between two different levels of hospitals[5]. These differences were not only associated with geography, environment, climate, and customs; they were also associated with economic factors.

As the largest developing country in the world, China has a large number of local hospitals; these could be considered as bridges that connect community hospitals and provincial hospitals. Therefore, if we are to achieve the '0by $25^{\prime}$ ' goal, it is very pertinent to carry out an in-depth analysis of the characteristics of AKI in local hospitals, enhance the recognition rate of AKI patients in local hospitals, and improve the prognosis of AKI patients. To this end, we depicted a range of etiological, clinical, and therapeutic factors, in AKI patients from local hospitals by economic levels and geographic regions. We also explored the risk factors associated with in-hospital mortality among the patients.

\section{Methods}

\section{Study design and participants}


The data we analyzed in this study were extracted from the 2013 nationwide cross-sectional survey of AKI conducted by the ISN Acute Kidney Failure (AKF) Oby25 China Consortium[5]. Data from the 22 local hospitals were used in the following analsyis. The detailed study design and procedures have been described in previous publications[5]. Briefly, adult patients ( $\geq 18$ years old) who were suspected to have AKI based on changes in serum creatinine level were identified by using the hospital-based Laboratory Information System (LIS). Due to the heavy workload, and considering the seasonal factors affecting AKI, the inpatient medical records of two single months (January and July) in 2013 were reviewed to define AKI patients. The diagnosis of AKI was confirmed according to the 2012 KDIGO (Kidney Disease: Improving Global Outcomes) diagnostic criteria: serum creatinine level increased by $0.3 \mathrm{mg} / \mathrm{dl}$ within 48 hours, or increased by $50 \%$ of baseline level within 7 days (regardless of urine output)[6]. In order to avoid misdiagnosis of AKI patients who did not have repeated serum creatinine assay within 7 days or those who had a recovering community-acquired AKI, an expanded diagnostic criteria was used to which referred to an increase or decrease in serum creatinine level by at least $50 \%$ of the baseline of the lowest or the highest serum creatinine levels during hospitalization[5]. Patients were excluded if they had stage 5 chronic kidney disease (CKD5), had undergone nephrectomy or kidney transplantation, had a peak value of serum creatinine $<53$ $\mu \mathrm{mol} / \mathrm{L}$, or met the diagnostic criteria but the change in serum creatinine was not caused by AKI.

Using the National Bureau of Statistics of the People's Republic of China, we obtained the per capita gross domestic product (pcGDP) of different regions in 2013. This was then used to divide the hospitals into three groups via tertiles of pcGDP levles, representing hospitals from economically underdeveloped region, moderately economic region, and economically developed region respectively. The hospitals were also stratified into four groups of geographic regions, which included Northwest China, North China, South China, and Southwest China.

\section{Procedure and definition of clinical indicators}

By reviewing patients' medical records, we were able to collate a range of information relating to sociodemographic status, comorbidities (hypertension, diabetes, and coronary heart disease, etc), underlying diseases, factors that may cause renal hypoperfusion (vomiting/diarrhea/excessive sweating/burns over large areas, or heart-kidney syndrome, etc), nephrotoxic drugs (antibiotics, contrast agents, non-steroidal anti-inflammatory drugs, and Chinese medicine etc), environmental toxins, other nephrotoxins (pesticides, snake bites, and bee stings, etc), and critical diseases (sepsis, multiple organ failure, stroke, shock, disseminated intravascular coagulation (DIC), acute respiratory distress syndrome, tracheal intubation ventilator-assisted ventilation, and advanced malignant tumors). In addition, we also recorded AKI classification (pre-renal, intrinsic-renal, post-renal), peak AKI stage (1 to 3 ), the timing of renal replacement therapy (RRT), all-cause in-hospital mortality, renal function recovery, and cost on discharge.

We also considered strict indications for RRT treatment[7, 8]. According to the KDIGO guidelines, patients with any of the highrisk/medium-risk factors were indicated for RRT treatment. Timely RRT treatment was defined as a patient receiving RRT when showing clear indications for treatment. The RRT treatment rate was defined as the ratio of the number of patients receiving RRT treatment to the number of patients who had indications for RRT treatment.

Full recovery of renal function implied that the serum creatinine had decreased to below the threshold value or the baseline value; partial recovery implied that the serum creatinine had decreased by more than $25 \%$ of the peak value but was still higher than the threshold value or the baseline value, and that patients were able to stop dialysis. Failure to recover implied that the patient relied on dialysis or that the decrease in serum creatinine was less than $25 \%$ of the peak value. Treatment withdrawal implied that the patient's condition remained serious but the patient was giving up on continual hospitalization because of economic or personal factors.

We considered that physicians had recognized AKI in their patients when the medical records referred to increased levels of serum creatinine, concerns relating to reduced renal function, reduced urine output, or when there had been adjustments of related treatment strategy. Timely recognition was defined as when a patient was diagnosed with AKI by the physician-in-charge within 3 days of the occurrence of AKI and before the AKI progressed to a more severe stage; otherwise, the case was classified as delayed recognition. AKI was considered as non-recognized when the physician-in-charge did not recognize that patients met the diagnostic criteria for AKI during hospitalization.

\section{Statistical analysis}

The detection rates of AKI were considered as a ratio of the number of detected AKI cases to the number of admissions during the same period. Continuous data are presented as means with standard deviation (SD) or as medians with interquartile ranges, as 
appropriate. Categorical variables are presented as proportions. A range of data were analyzed for AKI in local hospitals in different geographical regions and in association with different levels of economic development, including clinical characteristics, diagnoses, treatments, and prognoses. Comparisons among groups were carried out using one-way analysis of variance (ANOVA) or the KruskalWallis test for continuous variables. The chi-squared test was used to test categorical variables. Relevant covariates that may show association with all-cause in-hospital mortality were analyzed using logistic regression. We first conducted univariate analysis covering a range of covariates. Then, those with significant associations with in-hospital mortality were included in the multivariable analysis.Odds ratios (ORs) with $95 \%$ confidence intervals (Cls) and $P$ values based on Wald chi-squared test were reported. Analyses were performed with IBM SPSS software (version 22.0; IBM Institute Inc). All $P$ values are two-sided and a value of $P<0.05$ was considered to be statistically significant.

\section{Results}

\section{The detection rates of $\mathrm{AKI}$ in local hospitals from regions of different economic levels}

Between January and July 2013, a total of 116,788 patients were admitted to the 22 local hospitals included in our study. 1,942 patients were identified as AKI using the expanded criteria, corresponding to a detection rate of $1.66 \%$, including 790 cases $(0.68 \%)$ that were identified by the KDIGO criteria. These regions were divided into three groups by tertiles according to pcGDP levels: pcGDP Tertile 1 (underdeveloped regions), pcGDP Tertile 2 (moderately devoloped regions), and pcGDP Tertile 3 (developed regions). A comparison of detection rates among the three pcGDP groups showed that the detection rate for AKI in the pcGDP Tertile 1 group was the lowest ( $0.49 \%$ and $1.24 \%$ according to the KDIGO and expanded criteria, respectively). We also found that the recognition rates for AKI (including timely-recognition and delayed recognition) in the pcGDP Tertile1 and pcGDP Tertile 2 groups were significantly lower than that in the pcGDP Tertile 3 group $(26.38 \%$ and $24.32 \%$ vs $32.98 \%, P=0.001$, respectively). There were also significant differences in the detection rate for AKI when compared between different regions with different economic levels, as shown in Table 1.

\section{Characteristics of patients with AKI in local hospitals from regions of different economic levels}

The age of the 1,942 AKI patients identified in local hospitals was $64.0 \pm 17.2$ years, with more elder patients in developed areas. Male patients were the majority of AKI cases in all regrions. Of the 1,942 AKI patients, 1,070 (55.1\%) cases were classified as having prerenal AKI, 468 (24.1\%) cases as intrinsic-renal AKI, 198 (10.2\%) cases as post-renal AKI, and the remaining $206(10.6 \%)$ patients could not be clearly classified. With the exception of intrinsic-renal AKI, there were significant differences in the proportion of other AKI classifications across the different pcGDP groups (thus representing differences in accordance to economic level) (Table 2). The most common causes that might contribute to the development of AKI were low renal perfusion (78.0\%), drug factors (69.9\%), and other critical illness (38.1\%). Compared with moderately developed regions and developed regions, the proportion of AKI cases caused by nephrotoxic drugs was the lowest in underdeveloped regions ( $59.7 \%$ vs $72.7 \%$ vs $72.7 \%, P<0.001$ ) (Table 2 ).

Patients in local hospitals from underdeveloped regions had the highest proportion of patients with unclassified AKI (12.9\% vs $11.5 \%$ vs $7.4 \%, P=0.010)$ or reaching AKI stage $3(37.9 \%$ vs $25.1 \%$ vs $25.7 \%, P<0.001)$ compared to patients from moderately developed or devleoped regions. Compared with underdeveloped regions and developed regions, the proportion of AKI cases of referral to nephrology providers was the lowest in moderately developed $(16.9 \%$ vs $22.3 \%$ vs $22.8 \%, P=0.007)$. There was no difference in the proportion of patients in ICU, with indicators for RRT, or receiving RRT treatment, when compared among different pcGDP tertile groups. The proportion of patients who had not been recognized with AKI on time (including non-recognition and delayed recognition) was higher in local hospitals from underdeveloped and moderately developed regions than that in developed regions $(P<0.001)$. Local hospitals in underdeveloped regions had the highest proportion of patients with unrecovered renal function $(34.4 \%$ vs $29.4 \%$ vs $31.6 \%$, $P=0.04)$ and the highest mortality rate $(15.5 \%$ vs $10.3 \%$ vs $7.0 \%, P<0.001)$ compared to those from moderately developed or devleoped regions. There was no significant difference in the duration of hospital stays or treatment costs of AKI patients when compared among local hospitals from different economic levels (Table 2).

\section{The characteristics of AKI patients in local hospitals from different geographic regions}

The frequency of AKI patients in South China and Southwest China was noticeably higher than those in other geographic regions, regardless of whether the diagnoses were based on KDIGO criteria, expanded criteria, AKI recognition rate, or nephrology referral. When 
analyzing the classification of AKI, we found that pre-renal AKI was more common in the Western regions (including the Southwest and Northwest; $63.9 \%$ and $62.5 \%$, respectively) than that in North $(56.5 \%)$ and South $(51.5 \%)$ regions $(P<0.001)$. Etiological analysis showed that injury factors other than renal hypoperfusion to the kidneys differed significantly across different geographic regions. Northwest and Southwest regions had higher proportion of patients with potential nephrotoxic drugs use $(75.0 \%$ and $76.3 \%$, respectively). The Southwest region had the most patients with environmental toxin-induced AKI compared to other regions ( $10.0 \%$ vs $0.0 \% \sim 1.9 \%, P<0.001)$. AKI caused by sepsis or surgical factors was more common in South China. The underlying diseases also varied across different geographic regions. AKI patients with diabetes, cardiovascular disease, and cerebrovascular disease, were more frequently found in North China. Northwest China had the highest proportion of AKI patients with hypertension. AKI patients with basic chronic kidney disease and malignancies were most common in South China (Table 3).

\section{Risk factors affecting in-hospital mortality of AKI patients in local hospitals}

Altogether 1,913 patients with AKI had information on mortality during hospitalization, and the rate of all-cause in-hospital mortality was $10.5 \%(200 / 1,913)$. In the univariate analysis, age, sex, geographic region, pcGDP, AKI classification, injury factors, AKI stage, delayed recognition, staying in ICU, indication for RRT, RRT rate, comorbidity of CEVD and malignancy were associated with the outcome (all $P$ values $<0.05$ ). We included the above variables in the multivariable analysis and found that age, sex, geographic region, staying in ICU, AKI stage, injury factors, recognition of AKI and pcGDP levels were still associated with the outcome (all $P$ values $<0.05$ ). Among them, advanced age ( $\geq 80$ versus $18-39, \mathrm{OR}=2.34,95 \% \mathrm{Cl}: 1.21-4.56$ ), male gender (versus female, $\mathrm{OR}=1.60$, 95\%Cl: $1.10-2.33)$, staying in ICU (OR $=1.61,95 \% \mathrm{Cl}: 1.08-2.40)$, higher AKI stages (stage 2 versus stage $1, \mathrm{OR}=1.79,95 \% \mathrm{Cl}: 1.13-$ 2.83; stage 3 versus stage $1, \mathrm{OR}=3.59,95 \% \mathrm{Cl}: 2.28-5.67)$, using nephrotoxic drugs $(\mathrm{OR}=2.02,95 \% \mathrm{Cl}: 1.28-3.20)$, being with other critical illness $(\mathrm{OR}=6.31,95 \% \mathrm{Cl}: 3.93-10.15)$ and delayed recognition (versus timely recognition, $\mathrm{OR}=2.77,95 \% \mathrm{Cl}: 1.47-5.22)$ were associated with increased in-hospital mortality, while in hospitals from South China ( $\mathrm{OR}=0.43,95 \% \mathrm{Cl}: 0.27-0.71)$ or from regions with the highest pcGDP levels (tertile 3 versus tertile $1, \mathrm{OR}=0.44,95 \% \mathrm{Cl}: 0.25-0.78$ ) were associated with decreased in-hospital mortality (Table 4).

\section{Discussion}

AKI is a common public health problem that can adversely affect a patient's quality of life and even lead to death. According to the literature, there are significant differences in the diagnosis and treatment of AKI when compared between developed and developing countries[2, 9-13], socioeconomic status within the same country[14]. However, previous studies were mainly from academic hospitals, worldwidely there has been limited data regarding AKI patients in local hospitals. In the current study, we identified 1,942 AKI patients that were admitted to 22 local hospitals from a database of a national survey on AKI in China[5]. The detection rates, the clinical characteristics, and the stutas of diagnosis and treatment of AKI were analyzed and compared in the local hospitals at different economic levels and from different geographic regions.

The detection rate of AKI according to the expanded KDIGO criteria was $1.66 \%$ in the 1,942 patients from local hospitals, which was significantly lower than of the academic hospitals (2.20\%) from the same national survey[5]. We next stratified the local hospitals according to their regional economic levels and found that the detection rate of AKI in underdeveloped regions (1.24\%) was significantly lower than that in other regions (1.57\%-2.04\%). These discrepancies could be attributed to less serial testing for serum creatinine during patients' hospitalization in local hospitals especially in those from underdeveloped regions. More importantly, our data showed that AKI recognition rates also varied according to regional economic levels, with local hospitals from the most developed regions having the highest rates of both AKI recognition and timely reconition compared to those from moderately developed or underdevleoped regions. Meanwhile, patients in local hospitals from underdeveloped regions had the highest proportion of cases with unclassified AKI, which reflects the ability of AKI etiology identification by the physicians, or reaching stage 3 AKI, which could be, at least partially, due to the delayed recognition of AKI, compared to patients from more developed regions. These data indicate a more severe situation of AKI diagnosis in local hospitals from underdeveloped regions in China, which could be due to the less social security covering, the lack of nephrologist specialty, and the less understanding of the significance of AKI in clinical disciplinaries. More attention and efforts should be paid to increase the knowledge and recognition ability of AKI in local hospitals, especially in underdeveloped regions in China.

Our analysis showed that the causes of AKI in local hospitals were related to geographical regional factors. For example, AKI patients from South China had more cases related to sepsis, critical illness, and surgery when compared to patients from other regions. They also had higher proportion of AKI cases having baseline chronic kidney disease and maligancy. Patients from Southwest China had the highest frequency of AKI relavent to neprhotoxic insults, including both neprhotoxic medications and enviromental toxins among 
patients of the four geographic regions. AKI patients from North China had the highest proportion of cases having diabetes, cardiovascular diseases and cerebrovascular diseases. It seems that AKI patients from Northwest China had a very high rate of renal hypoperfusion and a much higher proportion of cases having baseline hypertension compared to AKI patients from other regions. These discrepancies in the etiology and baseline commorbidities among AKI patients from different geographic regions could be, at least partialy, related to the local climates, for example the subtropical monsoon climate in Southwest China which favors the exposure to enviromental nephrotoxins[10], and local dietary habits, for example the high salt diet in Norh and Northwest China which is an important risk factor to the development of hypertension and cardio-cerebrovascular diseases. Therefore, depicting the etiological profile of AKI in each region is of critical importance when making local preventing and treament strategy for AKI.

With the aging of the population, more and more elderly patients are hospitalized for treating various diseases, and many of them are admitted in local hospitals. Our data showed that over 80 years-of-age was an independent risk factor for in-hospital death of AKI patients in local hospitals, which reflects the big challenge in managing elder inpatients with complicated co-morbidities. Other independent risk factors for mortality included ICU admission, of critical illness, of a higher severity of AKI, and of delayed recognition. These results are consistent with other literature reports. In addition, our data showed that AKI patients in South China and from ecnomically developed regions represented independent protective factors against in-hospital death. This further proved that the level of economic development was associated with the outcome of AKI patients in local hospitals. In underdeveloped regions in China, training of medical personnel to raise awareness, to be able to quickly identify patients suspected of AKI, and to provide clinical treatment for AKI patients is essential.

The present study described the composition and the present diagnostic and treatment status of AKI in local hospitals from different economic and geographic regions of China. As far as we know, there have been few studies focusing on AKI in local hospitals across the world. However, our study had some limitations that need to be considered. Firstly, we referred to the $2012 \mathrm{KDIGO}$ diagnostic criteria for AKI. Because this was a retrospective study, most of the patients' records relating to urine output were absent. Consequently, we were reliant on the dynamic evolution of serum creatinine; there is a risk of missed diagnosis using this method. Secondly, local hospitals did not monitor serum creatinine often enough, which would increase the chances of missed diagnosis. Thirdly, due to the heavy workload involved in this study, we chose January and July as representatives of the winter and summer seasons. However, it is possible that these months were not necessarily reflective of these seasons and thus might result in sampling error. Nevertheless, this study provides objective data relating to the epidemiology of AKI inpatients in local hospitals in developing countries.

In conclusion, the etiology and presentation of AKI in local hospitals vary among regions with different economic and geographic features. Depicting a full picture of local AKI is of great importance to make regional preventive and treatment strategies.

Strengthening the knowledge of AKI, closely monitoring of renal function, initiating early reference to nephrologists, and improving the treatment of critical patients would improve the diangosis, management, and the prognosis of AKI patients. Moreover, the improvement of healthcare system for supporting AKI treatment in local hospitals, especially in underdeveloped regions are urgently needed.

\section{Abbreviations}

AKI: acute kidney injury; ISN: International Society of Nephrology; AKF: Acute Kidney Failure; LIS: Laboratory Information System; KDIGO: Kidney Disease; Improving Global Outcomes; CKD: chronic kidney disease; pcGDP: per capita gross domestic product; DIC: disseminated intravascular coagulation; RRT: renal replacement therapy; SD: standard deviation; ANOVA: analysis of variance; ORs: Odds ratios; Cls: confidence intervals; ICU: intensive care unit; CLD: chronic liver disease; CPD: chronic pulmonary disease; CEVD: cerebrovascular disease; CVD: cardiovascular disease.

\section{Declarations}

\section{Acknowledgements}

We thank all the group members of ISN AKF 0 by 25 China Consortium for their contributions.

\section{Funding}


This study was supported by grants from the National Natural Science Foundation of China (No.91742205, No.81625004, No.81860129), the Beijing Young Scientist Program (BJJWZYJH01201910001006), and Peking University Clinical Scientist Program by the Fundamental Research Funds for the Central Universities.

\section{Availability of data and materials}

The datasets used and/or analysed during the current study available from the corresponding author on reasonable request.

\section{Ethics approval}

This study was performed in accordance with the Declaration of Helsinki, approved by the Ethics Committee of Peking University First Hospital, and the requirement for informed consent was waived due to the retrospective nature of the study (2014[729]).

\section{Consent to participate}

Not applicable.

\section{Conflict of Interest Statement}

The authors have no conflicts of interest to declare.

\section{Consent for publication}

Not applicable.

\section{Authors' contributions}

Lihua Wu collected and analyzed data, and drafted the manuscript. Menghua Chen and Li Yang conceived, designed, and organised the study, interpreted the results, and revised the manuscript. Jinwei Wang helped analyse the data. All authors contributed to collecting the data on site.

\section{Author details}

1Department of Nephrology, General Hospital of Ningxia Medical University, Ningxia, China. ${ }^{2}$ Renal Division, Peking University First Hospital, Beijing, China. ${ }^{3}$ School of Basic Medical Sciences, Ningxia Medical University, Ningxia, China.

\section{References}

1. Lewington AJ, Cerda J, Mehta R L. Raising awareness of acute kidney injury: a global perspective of a silent killer. Kidney Int 2013,84:457-467.

2. Susantitaphong P, Cruz DN, Cerda J, Abulfaraj M, Alqahtani F, Koulouridis I, et al. World Incidence of AKI: A Meta-Analysis. Clin J Am Soc Nephro 2013, 8:1482-1493.

3. Remuzzi G, Horton R. Acute renal failure: an unacceptable death sentence globally. The Lancet 2013, 382: 2041-2042.

4. Lameire NH, Bagga A, Cruz D, Maeseneer JD, Endre Z, Kellum JA, et al. Acute kidney injury: an increasing global concern. The Lancet 2013, 382: 170-179.

5. Yang L, Xing GL, Wang L, Wu YG, Li SH, Xu G, et al. Acute kidney injury in China: a cross-sectional survey. The Lancet 2015. 386 : 1465-1471.

6. Kidney Disease: Improving Global Outcomes (KDIGO) Acute Kidney Injury Work Group. KDIGO Clinical practice guideline for acute kidney injury. Kidney Int Supp/2012. 2: 1-138.

7. Palevsky PM. Renal Replacement Therapy in Acute Kidney Injury. Adv Chronic Kidney Dis 2013, 20: 76-84.

8. Ricci Z, Ronco C. Timing, dose and mode of dialysis in acute kidney injury. Curr Opin Crit Care 2011, 17: 556-561.

9. Rewa O, Bagshaw SM. Acute kidney injury-epidemiology, outcomes and economics. Nat Rev Nephrol 2014, 10: 193-207.

10. Chertow GM, Burdick E, Honour M, Bonventre JV, Bates DW. Acute Kidney Injury, Mortality, Length of Stay, and Costs in Hospitalized Patients. J Am Soc Nephrol 2005, 16: 3365-3370. 
11. Uchino S, Bellomo R, Goldsmith D, Bates S, Ronco C. An assessment of the RIFLE criteria for acute renal failure in hospitalized patients. Crit Care Med 2006, 34: 1913-1917.

12. Nash K, Hafeez A, Hou S. Hospital-acquired renal insufficiency. Am J Kidney Dis 2002, 39: 930-936.

13. Thomas M, Sitch A, Dowswell G. The initial development and assessment of an automatic alert warning of acute kidney injury. Nephrol Dial Transp/ 2011, 26: 2161-2168.

14. Hounkpatin HO, Fraser SDS, Johnson MJ, Harris S, Uniacke M, Roderick PL. The association of socioeconomic status with incidence and outcomes of acute kidney injury. Clinical Kidney Journal 2020, 13: 245-252.

\section{Tables}

Table 1. Detection rates of AKI in local hospitals from regions of different economic levels

\begin{tabular}{ccccc}
\hline Total & pcGDP & pcGDP & pcGDP & Tertile 2 \\
$(\mathrm{n}=1942)$ & Tertile 1 & $(\mathrm{n}=958)$ & $(\mathrm{n}=567)$
\end{tabular}

\begin{tabular}{llllll}
\hline pcGDP & $22250.16(17801.02,34606.30)$ & $15988.34(13031.04,15988.34)$ & $22250.16(19701.78,23849.80)$ & $34606.30(34606.30$, & --
\end{tabular}

$54058.22)$

\begin{tabular}{|c|c|c|c|c|c|}
\hline Screened cases & 116788 & 33660 & 47028 & 36100 & -- \\
\hline $\begin{array}{l}\text { AKI detection } \\
\text { (KDIGO } \\
\text { criteria) Cases }\end{array}$ & $790(0.68 \%)$ & $164(0.49 \%)$ & $385(0.82 \%)^{\square}$ & $241(0.67 \%)^{0]}$ & $<0.001$ \\
\hline $\begin{array}{l}\text { AKI detection } \\
\text { (expanded } \\
\text { criteria) }\end{array}$ & $1942(1.66 \%)$ & $417(1.24 \%)$ & $958(2.04 \%)^{\square}$ & $567(1.57 \%)^{0]}$ & $<0.001$ \\
\hline \multicolumn{6}{|l|}{ Cases } \\
\hline $\begin{array}{l}\text { AKI recognition } \\
\text { (KDIGO } \\
\text { criteria) Cases }\end{array}$ & $530(27.29 \%)$ & $110(26.38 \%)$ & $233(24.32 \%)$ & $187(32.98 \%)^{0}$ & 0.001 \\
\hline $\begin{array}{l}\text { Region } \\
\text { recognition } \\
\text { (KDIGO } \\
\text { criteria) Cases }\end{array}$ & & & & & $<0.001$ \\
\hline Northwest & $6(0.16 \%)$ & $6(0.16 \%)$ & $0(0.00 \%)$ & $0(0.00 \%)$ & \\
\hline North & $93(0.29 \%)$ & $61(0.35 \%)$ & $11(0.44 \%)$ & $21(0.17 \%)$ & \\
\hline South & $316(0.51 \%)$ & $21(0.34 \%)$ & $129(0.40 \%)$ & $166(0.70 \%)$ & \\
\hline Southwest & $115(0.61 \%)$ & $22(0.36 \%)$ & $93(0.74 \%)$ & $0(0.00 \%)$ & \\
\hline
\end{tabular}

Abbreviations: AKI, acute kidney injury; pcGDP, per capita gross domestic product; KDIGO, Kidney Disease: Improving Global Outcomes.

${ }^{\square} P<0.05$ between pcGDP Tertile 2 and pcGDP Tertile $1 ;{ }^{\natural} P<0.05$ between pcGDP Tertile 3 and pcGDP Tertile1.

Table 2. The characteristics of patients with AKI in local hospitals from regions of different economic levels 


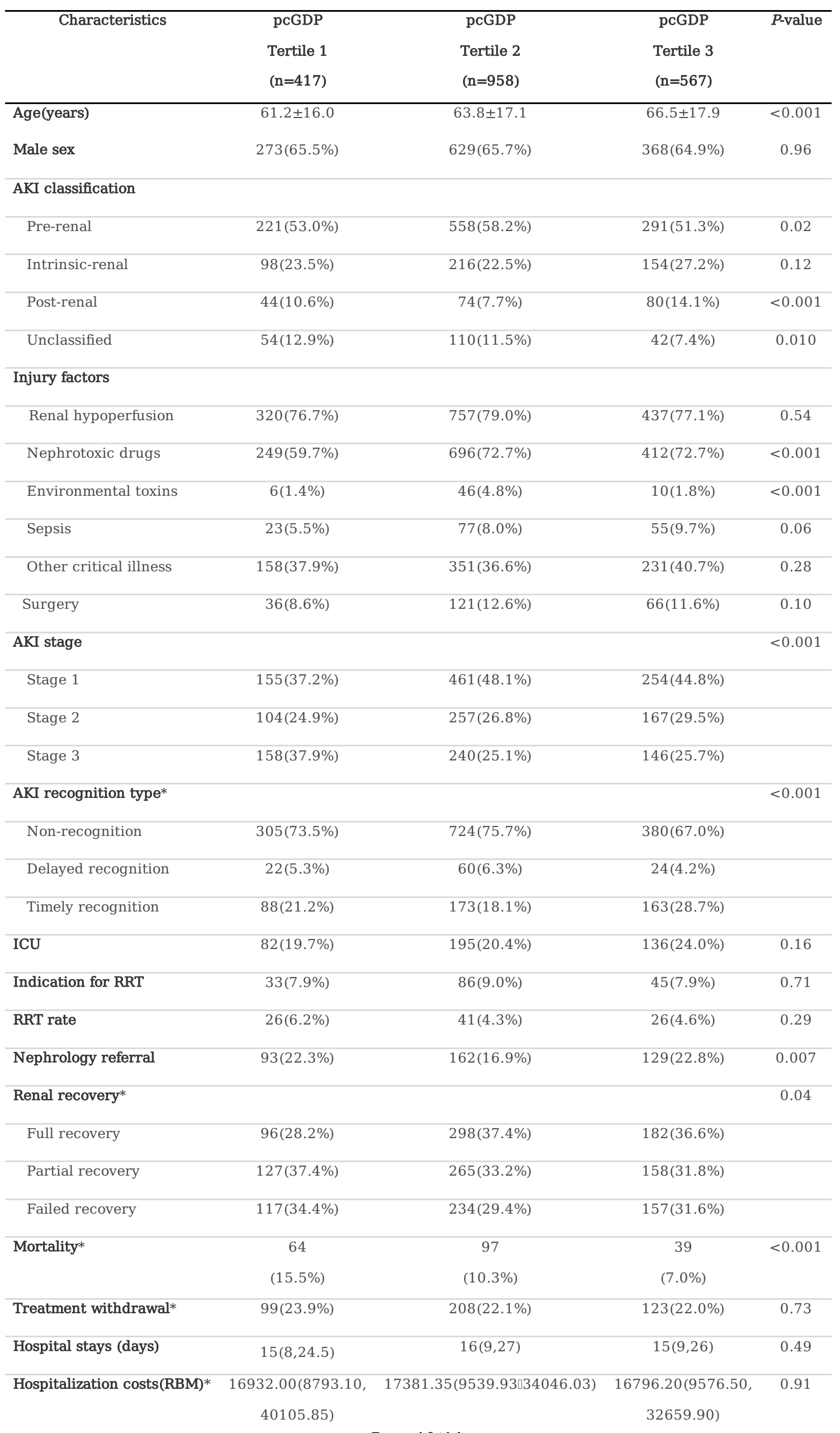

Page 10/14 


\begin{tabular}{|c|c|c|c|c|}
\hline Comorbidity & & & & \\
\hline Diabetes & $64(15.3 \%)$ & $171(17.8 \%)$ & 104(18.3\%) & 0.43 \\
\hline Hypertension & $161(38.6 \%)$ & $352(36.7 \%)$ & $269(47.4 \%)$ & $<0.001$ \\
\hline Pre-existing CKD & $70(16.8 \%)$ & $186(19.4 \%)$ & $160(28.3 \%)$ & $<0.001$ \\
\hline Cardiovascular disease & $145(34.8 \%)$ & $360(37.6 \%)$ & $238(42.0 \%)$ & 0.06 \\
\hline CLD & $25(6.0 \%)$ & $70(7.3 \%)$ & $50(8.8 \%)$ & 0.24 \\
\hline CPD & $35(8.4 \%)$ & $148(15.4 \%)$ & $108(19.0 \%)$ & $<0.001$ \\
\hline CEVD & $76(18.2 \%)$ & $160(16.7 \%)$ & $115(20.3 \%)$ & 0.21 \\
\hline Anemia & $18(4.3 \%)$ & $236(24.6 \%)$ & $130(22.9 \%)$ & $<0.001$ \\
\hline Other CVD & $54(12.9 \%)$ & 188(19.6\%) & $135(23.8 \%)$ & $<0.001$ \\
\hline Malignancy & $45(10.8 \%)$ & 134(14.0\%) & $95(16.8 \%)$ & 0.03 \\
\hline
\end{tabular}

Abbreviations: AKI, acute kidney injury; pcGDP, per capita gross domestic product; ICU, intensive care unit; RRT, renal replacement therapy; CKD, chronic kidney disease; CLD, chronic liver disease; CPD, chronic pulmonary disease; CEVD, cerebrovascular disease; CVD, cardiovascular disease.

*Date missing for mortality in 29 cases(3 in pcGDP Tertile1 vs 18 in pcGDP Tertile2 vs 8 in pcGDP Tertile3), for treatment withdrawal in 29 cases(3 vs 18 vs 8 ), for AKI recognition type in 3 cases( 2 vs 1 vs 0 ), for renal recovery in 308 cases(77 vs 161 vs 70 ), and hospital costs in 279 cases( 23 vs 212 vs 44).

Table 3. The characteristics of AKI patients in local hospitals from different geographic regions 


\begin{tabular}{|c|c|c|c|c|c|}
\hline & $\begin{array}{c}\text { Northwest } \\
(n=24)\end{array}$ & $\begin{array}{c}\text { North } \\
(n=405)\end{array}$ & $\begin{array}{c}\text { South } \\
(\mathrm{n}=1134)\end{array}$ & $\begin{array}{l}\text { Southwest } \\
(n=379)\end{array}$ & $P$-value \\
\hline Age(years) & $61.4 \pm 19.9$ & $64.1 \pm 16.0$ & $64.6 \pm 17.5$ & $62.5 \pm 17.4$ & 0.17 \\
\hline Male sex & $15(62.5 \%)$ & $260(64.2 \%)$ & $741(65.3 \%)$ & $254(67.0 \%)$ & 0.85 \\
\hline AKI detection (KDIGO criteria) Cases & $7(0.19 \%)$ & $172(0.53 \%)$ & $491(0.80 \%)$ & $120(0.64 \%)$ & $<0.001$ \\
\hline AKI detection (expanded criteria) & $24(0.64 \%)$ & $405(1.24 \%)$ & $1134(1.84 \%)$ & $379(2.01 \%)$ & $<0.001$ \\
\hline \multicolumn{6}{|l|}{ Cases } \\
\hline AKI recognition & $6(25.00 \%)$ & $93(22.96 \%)$ & $316(27.87 \%)$ & $115(30.34 \%)$ & $<0.001$ \\
\hline Nephrology referral & $3(12.5 \%)$ & $72(17.8 \%)$ & $212(18.7 \%)$ & $97(25.6 \%)$ & 0.01 \\
\hline \multirow[t]{3}{*}{ pcGDP } & 2326.64 & 15988.34 & 22250.16 & 23849.80 & $<0.001$ \\
\hline & $(2326.64 \llbracket 2326.64)$ & $(15988.34,17801.02)$ & (19701.78, & $(23849.80 \square 23849.80)$ & \\
\hline & & & $34606.30)$ & & \\
\hline \multicolumn{6}{|l|}{ AKI classification } \\
\hline Pre-renal & $15(62.5 \%)$ & $229(56.5 \%)$ & $584(51.5 \%)$ & $242(63.9 \%)$ & $<0.001$ \\
\hline Intrinsic-renal & $6(25.0 \%)$ & $96(23.7 \%)$ & $297(26.2 \%)$ & $69(18.2 \%)$ & 0.02 \\
\hline Post-renal & $1(4.2 \%)$ & $30(7.4 \%)$ & $124(10.9 \%)$ & $43(11.3 \%)$ & 0.13 \\
\hline Unclassified & $2(8.3 \%)$ & $50(12.4 \%)$ & $129(11.4 \%)$ & $25(6.6 \%)$ & 0.04 \\
\hline \multicolumn{6}{|l|}{ Injury factors } \\
\hline Renal hypoperfusion & $23(95.8 \%)$ & $315(77.8 \%)$ & $883(77.9 \%)$ & $293(77.3 \%)$ & 0.21 \\
\hline Nephrotoxic drugs & $18(75.0 \%)$ & $250(61.7 \%)$ & $800(70.5 \%)$ & $289(76.3 \%)$ & $<0.001$ \\
\hline Environmental toxins & $0(0.0 \%)$ & $2(0.5 \%)$ & $22(1.9 \%)$ & $38(10.0 \%)$ & $<0.001$ \\
\hline Sepsis & $1(4.2 \%)$ & $13(3.2 \%)$ & $123(10.8 \%)$ & $18(4.7 \%)$ & $<0.001$ \\
\hline Other critical illness & $4(16.7 \%)$ & $161(39.8 \%)$ & $453(39.9 \%)$ & $122(32.2 \%)$ & 0.006 \\
\hline Surgery & $0(0.0 \%)$ & $36(8.9 \%)$ & $158(13.9 \%)$ & $29(7.7 \%)$ & $<0.001$ \\
\hline \multicolumn{6}{|l|}{ Comorbidity } \\
\hline Diabetes & $4(16.7 \%)$ & $94(23.2 \%)$ & $172(15.2 \%)$ & $69(18.2 \%)$ & 0.004 \\
\hline Hypertension & $15(62.5 \%)$ & $183(45.2 \%)$ & $485(42.8 \%)$ & $99(26.1 \%)$ & $<0.001$ \\
\hline Pre-existing CKD & $0(0.0 \%)$ & $75(18.5 \%)$ & $273(24.1 \%)$ & $68(17.9 \%)$ & 0.001 \\
\hline Cardiovascular disease & $10(41.7 \%)$ & $184(45.4 \%)$ & $420(37.0 \%)$ & $129(34.0 \%)$ & 0.006 \\
\hline CLD & $0(0.0 \%)$ & $23(5.7 \%)$ & $93(8.2 \%)$ & $29(7.7 \%)$ & 0.19 \\
\hline CPD & $4(16.7 \%)$ & $55(13.6 \%)$ & $161(14.2 \%)$ & $71(18.7 \%)$ & 0.14 \\
\hline CEVD & $1(4.2 \%)$ & $102(25.2 \%)$ & $196(17.3 \%)$ & $52(13.7 \%)$ & $<0.001$ \\
\hline Anemia & $1(4.2 \%)$ & $45(11.1 \%)$ & $270(23.8 \%)$ & $68(17.9 \%)$ & $<0.001$ \\
\hline Other CVD & $5(20.8 \%)$ & $64(15.8 \%)$ & $248(21.9 \%)$ & $60(15.8 \%)$ & 0.01 \\
\hline Malignancy & $0(0.0 \%)$ & $60(14.8 \%)$ & $173(15.3 \%)$ & $41(10.8 \%)$ & 0.03 \\
\hline
\end{tabular}

Abbreviations: AKI, acute kidney injury; KDIGO, Kidney Disease: Improving Global Outcomes; pcGDP, per capita gross domestic product; CKD, chronic kidney disease; CLD, chronic liver disease; CPD, chronic pulmonary disease; CEVD, cerebrovascular disease; CVD, cardiovascular disease.

Table 4. Analysis of risk factors affecting the in-hospital mortality rate of AKI patients in local hospitals* 


\begin{tabular}{|c|c|c|c|c|c|}
\hline & Univariate & & Multivari & & \\
\hline & Logistic Regression & Analysis & Logistic Regress & Analysis & \\
\hline & OR (95\% CI) & $P$-value & OR (95\% CI) & $P$-value & \\
\hline$\overline{\text { Age }}$ & & & & & \\
\hline $18-39$ & 1 (reference) & & 1 (reference) & & \\
\hline $40-59$ & $1.21(0.70-2.12)$ & 0.50 & $1.22(0.64-2.32)$ & 0.54 & \\
\hline $60-79$ & $0.83(0.48-1.44)$ & 0.51 & $0.87(0.46-1.65)$ & 0.68 & \\
\hline$\geq 80$ & $1.56(0.89-2.73)$ & 0.12 & $2.34(1.21-4.56)$ & 0.01 & \\
\hline Sex(male vs female) & $1.68(1.20-2.34)$ & 0.003 & $1.60(1.10-2.33)$ & 0.02 & \\
\hline Region & & & & & \\
\hline North & & & 1 (reference) & & \\
\hline South & $0.42(0.30-0.59)$ & $<0.001$ & $0.43(0.27-0.71)$ & 0.001 & 0.001 \\
\hline Northwest & $0.43(0.10-1.87)$ & 0.26 & $0.88(0.17-4.54)$ & 0.88 & 0.88 \\
\hline Southwest & $0.52(0.34-0.79)$ & 0.002 & $0.54(0.29-0.99)$ & 0.05 & 0.05 \\
\hline pcGDP & & & & & 0.02 \\
\hline Tertile $1(n=414)$ & 1 (reference) & & 1 (reference) & & \\
\hline Tertile $2(n=940)$ & $0.63(0.45-0.88)$ & 0.007 & $0.75(0.45-1.25)$ & 0.27 & \\
\hline Tertile $3(n=559)$ & $0.41(0.27-0.63)$ & $<0.001$ & $0.44(0.25-0.78)$ & 0.005 & \\
\hline AKI classification & & & & & \\
\hline Pre-renal & $1.12(0.83-1.50)$ & 0.47 & & & \\
\hline Intrinsic-renal & $1.47(1.07-2.02)$ & 0.02 & $1.06(0.72-1.57)$ & 0.77 & \\
\hline Post-renal & $0.39(0.19-0.76)$ & 0.006 & $0.50(0.23-1.07)$ & 0.07 & \\
\hline Unclassified & $0.56(0.32-1.01)$ & 0.05 & & & \\
\hline Injury factors & & & & & \\
\hline Renal hypoperfusion & $1.67(1.12-2.50)$ & 0.01 & $1.09(0.68-1.74)$ & 0.74 & \\
\hline Nephrotoxic drugs & $2.64(1.77-3.94)$ & $<0.001$ & $2.02(1.28-3.20)$ & 0.003 & \\
\hline Environmental toxins & $1.14(0.51-2.53)$ & 0.76 & & & \\
\hline Sepsis & $1.49(0.93-2.41)$ & 0.10 & & & \\
\hline Other critical illness & $9.42(6.37-13.93)$ & $<0.001$ & $6.31(3.93-10.15)$ & $<0.001$ & \\
\hline Surgery & $1.58(1.06-2.37)$ & 0.03 & $1.14(0.70-1.84)$ & 0.60 & \\
\hline AKI stage & & & & & \\
\hline Stage 1 & 1 (reference) & & 1 (reference) & & \\
\hline Stage 2 & $1.88(1.24-2.84)$ & 0.003 & $1.79(1.13-2.83)$ & 0.01 & \\
\hline Stage 3 & $4.24(2.94-6.12)$ & $<0.001$ & $3.59(2.28-5.67)$ & $<0.001$ & \\
\hline Delayed recognition vs Timely recognition & $2.59(1.51-4.43)$ & 0.001 & $2.77(1.47-5.22)$ & 0.002 & \\
\hline ICU & $3.13(2.30-4.25)$ & $<0.001$ & $1.61(1.08-2.40)$ & 0.02 & \\
\hline Indication for RRT & $3.16(2.13-4.69)$ & $<0.001$ & $1.74(0.94-3.22)$ & 0.08 & \\
\hline RRT rate & $2.43(1.43-4.12)$ & 0.001 & $0.81(0.35-1.83)$ & 0.61 & \\
\hline Nephrology referral & $1.16(0.81-1.66)$ & 0.41 & & & \\
\hline
\end{tabular}




\begin{tabular}{|c|c|c|c|c|}
\hline Diabetes & $0.93(0.63-1.38)$ & 0.72 & & \\
\hline Hypertension & $0.86(0.63-1.16)$ & 0.32 & & \\
\hline \multirow[t]{3}{*}{ Pre-existing CKD } & $0.74(0.50-1.11)$ & 0.14 & & \\
\hline & & & & \\
\hline & $1.25(0.93-1.68)$ & 0.14 & & \\
\hline CLD & $1.45(0.88-2.38)$ & 0.14 & & \\
\hline $\mathrm{CPD}$ & $1.13(0.76-1.68)$ & 0.55 & & \\
\hline CEVD & $1.54(1.09-2.18)$ & 0.01 & $1.44(0.94-2.20)$ & 0.10 \\
\hline Anemia & $1.36(0.96-1.92)$ & 0.08 & & \\
\hline Other CVD & $0.84(0.57-1.24)$ & 0.39 & & \\
\hline Malignancy & $2.72(1.94-3.83)$ & $<0.001$ & $1.56(0.99-2.45)$ & 0.05 \\
\hline
\end{tabular}

Abbreviations: AKI, acute kidney injury; pcGDP, per capita gross domestic product; ICU, intensive care unit; RRT, renal replacement therapy; CKD, chronic kidney disease; CLD, chronic liver disease; CPD, chronic pulmonary disease; CEVD, cerebrovascular disease; CVD, cardiovascular disease.

*1913 cases were included in the analysis after excluding 29 cases, which were missing the information for all-cause in-hospital mortality, and 3 for AKI recognition type. 